\title{
Observability and diagnostics in the X-ray band of shock-cloud interactions in supernova remnants
}

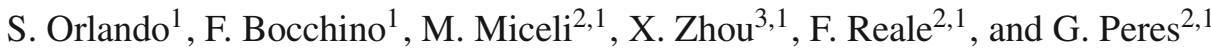 \\ 1 INAF - Osservatorio Astronomico di Palermo "G.S. Vaiana”, Piazza del Parlamento 1, 90134 Palermo, Italy \\ e-mail: orlando@astropa.unipa.it \\ 2 Dip. di Scienze Fisiche \& Astronomiche, Univ. di Palermo, Piazza del Parlamento 1, 90134 Palermo, Italy \\ 3 Department of Astronomy, Nanjing University, Nanjing 210093, PR China
}

Received 3 December 2009 / Accepted 13 January 2010

ABSTRACT

\begin{abstract}
Context. X-ray emitting features originating from the interaction of supernova shock waves with small interstellar gas clouds are revealed in many X-ray observations of evolved supernova remnants (e.g., Cygnus Loop and Vela), but their interpretation is not straightforward.

Aims. We develop a self-consistent method for the analysis and interpretation of shock-cloud interactions in middle-aged supernova remnants, which can provide the key parameters of the system and the role of relevant physical effects such as thermal conduction, without the need to perform ad-hoc numerical simulations and bother about morphology details.

Methods. We explore all the possible values of the shock speed and cloud density contrast relevant to middle-aged SNRs with a set of hydrodynamic simulations of shock-cloud interaction including the effects of thermal conduction and radiative cooling. From the simulations, we synthesize spatially and spectrally resolved focal-plane data as they would be collected with XMM-Newton/EPIC, an $\mathrm{X}$-ray instrument commonly used in these studies.

Results. We develop and calibrate two diagnostic tools, the first based on the mean photon energy versus count-rate scatter plot and the second on the spectral analysis of the interaction region, that can be used to highlight the effects of thermal conduction and to derive the shock speed in case of efficient conduction at work. These tools can be used to ascertain information from X-ray observations, without the need to develop detailed and ad-hoc numerical models for the interpretation of the data.
\end{abstract}

Key words. hydrodynamics - shock waves - ISM: clouds - ISM: supernova remnants - X-rays: ISM

\section{Introduction}

Supernova remnants (SNRs) are known to be invaluable laboratories for investigating the physical and chemical evolution of the galactic interstellar medium (ISM) and the mass distribution of the plasma in the Galaxy. Multiwavelength observations of evolved SNRs (e.g., Graham et al. 1995; Bocchino et al. 2000; Patnaude et al. 2002; Nichols \& Slavin 2004; Miceli et al. 2005) can be a useful tool for investigating the physics of SNRs, for instance the interaction of the remnants with inhomogeneities (clouds) in the ISM. However, this interaction involves many non-linear physical processes (e.g., radiative losses and thermal conduction), which complicate the analysis of the observations. An additional limitation comes from the superposition of different emitting regions along the line-of-sight (hereafter LoS) and, in most cases, the data interpretation is not unique.

A powerful approach to the data analysis is based on hydrodynamic and MHD simulations of the shock-cloud interaction, which takes into account the most relevant physical mechanisms (e.g., thermal conduction, radiative cooling), and on the comparison of the model results with observations. Previous studies (Orlando et al. 2005, 2006; Miceli et al. 2006; Orlando et al. 2008) were devoted to investigate, by means of numerical modeling, the interaction of SNR shock fronts with small interstellar gas clouds. In this paper we propose: i) to investigate the role of the different physical processes involved on the dynamics and energetics of the shocked cloud; and ii) to analyze accurately the SNR observations by comparing them with model results.

As part of this project, we previously investigated the role of thermal conduction and radiative cooling on the evolution of the shocked cloud in the unmagnetized limit. We explored two physical regimes in which each of the two physical processes in turn dominates (Orlando et al. 2005, hereafter Paper I) and found that, in general, the thermal conduction determines the evaporation of a fraction of the shocked cloud, forming a hot and tenuous gas phase (the corona) surrounding the cloud core. In the presence of an organized interstellar magnetic field, the thermal conduction is known to be inhibited across the magnetic field lines and the radiative cooling can be enhanced by magnetic plasma confinement. We studied the role played by the magnetic-field-oriented thermal conduction and the radiative cooling during the shockcloud interaction by considering different configurations of the magnetic field (Orlando et al. 2008). We found that the magnetized cases fall in-between the limit of completely suppressed thermal conduction and the unmagnetized limit with conduction.

Our numerical models were also used to predict the expected $\mathrm{X}$-ray emission from the shock-cloud interaction. We showed that the X-ray emitting structures do not trace the morphology of the flow structures originating from the shock-cloud interaction and that the shocked clouds are visible more easily during the early phases of their evolution (Orlando et al. 2006, hereafter Paper II). 
These significant efforts in modeling the shock-cloud interaction and its X-ray emission have not been superseded by the rigorous methodologies developed to compare X-ray observations of SNR shells and models. The high resolution instruments onboard XMM-Newton and Chandra have provided us with excellent images and spectra of SNRs, which are always much more complicated than the ideal cases treated in numerical simulations. Therefore, a straightforward comparison between models and observations is still difficult, and this tends to hamper our understanding of the details of the physical processes at the base of the X-ray radiation from SNR shells. Miceli et al. (2006) made the first attempt to accurately compare models with observations. They compared the X-ray observations of an isolated knot in the northern rim of the Vela SNR (Vela FilD, Miceli et al. 2005) with an ad-hoc hydrodynamic model; the comparison showed that the bulk of the X-ray emission in the knot originates in the cloud material heated by the transmitted shock front, but significant X-ray emission is also associated with the cloud material that evaporates in the intercloud medium, under the effect of the thermal conduction. While this strategy has proved to be effective, it is quite model-dependent, in the sense that it is based on an accurate yet restrictive morphological and spectral comparison, which may be time and resource consuming.

In contrast, the idea behind this paper is that the exploration of the parameter space of the shock-cloud model performed in Papers I and II, along with the extension presented here toward still unexplored values of the cloud density contrast, may be used to devise a quick and effective methodology for the interpretation of current generation X-ray satellite observations of shockcloud interactions, without the need to develop ad-hoc numerical models. Our intent is to provide easy-to-use recipes that allow us to extract from the data many of the key parameters governing the evolution of shocked clouds, by comparing with a set of model quantities normalized in such a way as to eliminate the dependence from unnecessary details, e.g., the exact morphology of the hit cloud. In particular, our scope includes devising a diagnostic tool capable of quickly assess whether the spectral results obtained in the interaction regions are dominated by thermal conduction, which is a physical effect whose contribution to the X-ray emission is modulated by the magnetic field and, therefore, remains uncertain.

The paper is organized as follows: Sect. 2 briefly describes the numerical setup, the physical parameters of the problem, and the method to synthesize, from the numerical simulations, X-ray observations as they would be performed by X-ray observatories; Sect. 3 presents the results of the numerical simulations; in Sect. 4, we describe the diagnostic tools devised in this paper and apply the methods, as an example, to X-ray observations reported in the literature; in Sect. 5, we draw our conclusions.

\section{Hydrodynamic modeling}

We model the three-dimensional interaction of a SNR shock front with an ISM cloud as described in Paper I, to which the reader is referred for more details. We summarize here the main model features. The cloud is assumed to be small compared to the curvature radius of the shock ${ }^{1}$ and in pressure equilibrium with the unperturbed isothermal and homogeneous ambient medium; we consider, therefore, a planar shock front and an isobaric cloud that is spherical for simplicity. The shock propagates with a Mach number $\mathcal{M} \gg 1$ in the ambient medium. The

1 This assumption is valid for a $1 \mathrm{pc}$ cloud in the middle-aged SNRs Vela and Cygnus Loop, whose shell has a radius $>10 \mathrm{pc}$. post-shock initial conditions are given by the strong shock limit (Zel'dovich \& Raizer 1966). The fluid is assumed to be fully ionized, and is regarded to be a perfect gas.

The plasma dynamics are described by solving numerically the time-dependent fluid equations of mass, momentum, and energy conservation (see Eqs. (1)-(5) in Paper I). The model takes into account both thermal conduction (Spitzer 1962) and radiative losses from an optically thin plasma (e.g., Raymond \& Smith 1977; Mewe et al. 1985; Kaastra \& Mewe 2000). The thermal conduction includes the free-streaming limit (saturation) on the heat flux (Cowie \& McKee 1977; Giuliani 1984; Borkowski et al. 1989; Fadeyev et al. 2002, and references therein). Our calculations also include a passive tracer associated with the cloud material that traces its motion during the evolution. A discussion of the assumptions of the model and their influence on the results is presented in Sect. 4.4.

The numerical code is FLASH (Fryxell et al. 2000), a multidimensional hydrodynamics code for simulating astrophysical plasmas that uses the PARAMESH (MacNeice et al. 2000) library for block-structured adaptive mesh refinement (AMR) and has been customized with numerical modules that model thermal conduction and optically thin radiative losses (see Paper I for details). The initial configuration, the boundary conditions, and the AMR setup of the simulations used here are the same as those adopted and discussed in Paper I.

As a reference case, we consider the $\mathcal{M}=50$ shock model described in Paper I; we then explore the parameter space by varying, alternatively, either the Mach number, $\mathcal{M}$, or the density contrast cloud/surrounding medium, $\chi$. In the reference model (RCm50c10), the unperturbed ambient medium is at a temperature $T_{\text {ism }}=10^{4} \mathrm{~K}$ and particle number density $n_{\text {ism }}=0.1 \mathrm{~cm}^{-3}$, and the spherical isobaric cloud has a radius $r_{\mathrm{cl}}=1 \mathrm{pc}$ and density contrast $\chi=10$ (particle number density $n_{\mathrm{cl}}=\chi n_{\mathrm{ism}}=$ $1 \mathrm{~cm}^{-3}$ ). The SNR shock front is planar at a Mach number $\mathcal{M}=$ 50 and temperature $T_{\mathrm{psh}}=4.7 \mathrm{MK}$. In the other simulations, the Mach number varies in the range $40 \leq \mathcal{M} \leq 60$ (corresponding to shock temperatures in the range $3 \mathrm{MK} \leq T_{\mathrm{psh}} \leq 7 \mathrm{MK}$ ) and the cloud density contrast is in the range $3 \leq \chi \leq 30$ (corresponding to a particle number density in the cloud in the range $0.3 \mathrm{~cm}^{-3} \leq n_{\mathrm{cl}} \leq 3 \mathrm{~cm}^{-3}$ ). We note that in Paper II, we studied the variation induced by a different choice of shock speed (we considered $\mathcal{M}=30$ and 50 at $\chi=10$ ). Here, we present for the first time the results for different density contrasts. These ranges are representative of most of the shock-cloud interaction regions observed in evolved SNRs (e.g., Vela, Cygnus Loop, and G296.5+10.0).

The effects of thermal conduction on the shocked cloud evolution were fully investigated in Papers I and II. Here, since we wish to develop a diagnostic for use in real X-ray observations, we compare the results of runs with this physical process (hereafter RC runs) with those of other runs without it (hereafter HY runs). As shown in a previous work (Orlando et al. 2008), shockcloud interactions in an organized interstellar magnetic field are in-between these two limits (i.e., HY and RC cases). A summary of all the simulations discussed in this paper is in Table 1, while Fig. 1 shows the simulations in the $\chi-\mathcal{M}$ parameter space. As discussed in Paper I (cf. Fig. 2 in Paper I), this plot can be used to evaluate whether radiative cooling has a comparable influence to thermal conduction in a given run. For example, the shock transmitted into the cloud is strongly radiative in runs $\mathrm{RCm} 40 \mathrm{c} 10$ and $\mathrm{RCm} 50 \mathrm{c} 30$. On the other hand, the thermal conduction dominates over the radiative losses in all the other cases (i.e., RCm50c03, RCm50c10 and RCm60c10): the cloud 
Table 1. Parameters of the simulated shock-cloud interactions.

\begin{tabular}{lcccccc}
\hline \hline Run & $\mathcal{M}^{a}$ & $\chi^{b}$ & $\begin{array}{c}w^{c} \\
{\left[\mathrm{~km} \mathrm{~s}^{-1}\right]}\end{array}$ & $\begin{array}{c}T_{\mathrm{psh}}{ }^{d} \\
{[\mathrm{MK}]}\end{array}$ & $\begin{array}{c}\tau_{\mathrm{cc}}{ }^{e} \\
{\left[10^{3} \mathrm{yr}\right]}\end{array}$ & $\begin{array}{c}\text { therm. } \\
\text { cond. }\end{array}$ \\
\hline HYm40c10 & 40 & 10 & 458 & 3.0 & 6.75 & no \\
HYm50c10 & 50 & 10 & 574 & 4.7 & 5.41 & no \\
HYm60c10 & 60 & 10 & 688 & 6.7 & 4.50 & no \\
RCm40c10 & 40 & 10 & 458 & 3.0 & 6.75 & yes \\
RCm50c10 & 50 & 10 & 574 & 4.7 & 5.41 & yes \\
RCm60c10 & 60 & 10 & 688 & 6.7 & 4.50 & yes \\
RCm50c03 & 50 & 03 & 574 & 4.7 & 2.96 & yes \\
RCm50c30 & 50 & 30 & 574 & 4.7 & 9.37 & yes \\
\hline
\end{tabular}

Notes. ${ }^{(a)}$ Shock Mach number. ${ }^{(b)}$ Density contrast cloud/ambient medium. ${ }^{(c)}$ Velocity of the SNR shock. ${ }^{(d)}$ Temperature of the postshock ambient medium. ${ }^{(e)}$ Cloud crushing time (Klein et al. 1994).

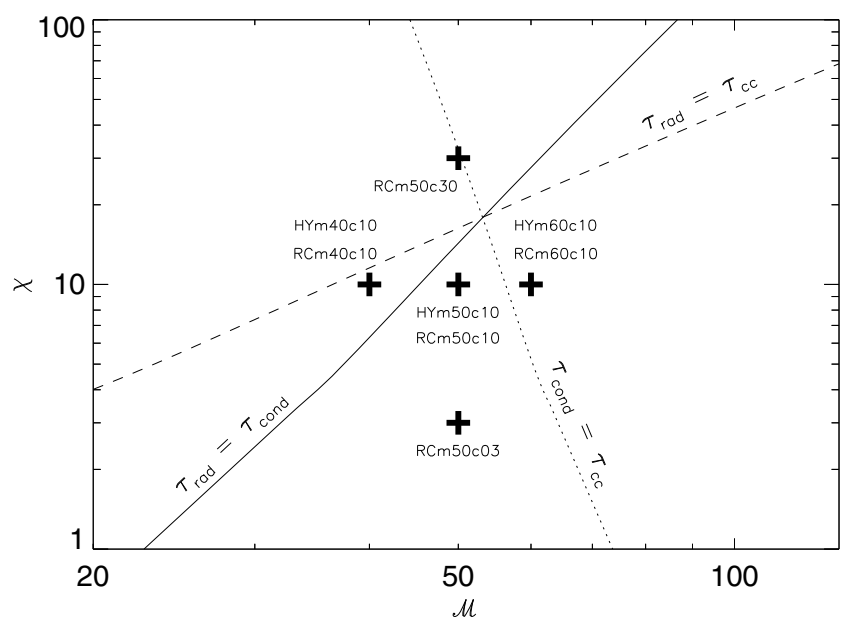

Fig. 1. $\chi-\mathcal{M}$ parameter space (adapted form Paper I). The lines are derived for length-scale $l=1 \mathrm{pc}$, and for an unperturbed ambient medium of temperature $T_{\text {ism }}=10^{4} \mathrm{~K}$ and particle number density $n_{\mathrm{ism}}=0.1 \mathrm{~cm}^{-3}$ (see Paper I for details): the solid line separates regions dominated by radiative cooling (on the left) from regions dominated by thermal conduction (on the right); the dashed line marks the density contrast, $\chi$, above which the cooling timescale $\tau_{\text {rad }}$ is shorter than the cloud crushing time $\tau_{\mathrm{cc}}$; the dotted line marks the values of $\chi$ above which the thermal conduction timescale $\tau_{\text {cond }}$ is shorter than $\tau_{\text {cc }}$. The parameter pairs explored are marked with crosses.

is expected to evaporate on a timescale comparable to (in $\mathrm{RCm} 50 \mathrm{c} 03$ and $\mathrm{RCm} 50 \mathrm{c} 10$ ) or shorter than (in RCm60c10) $\tau_{\mathrm{cc}}$.

\subsection{Synthesis of the $X$-ray observations}

The output of the numerical simulations is the evolution of temperature, density, and velocity of the plasma in the spatial domain. From the density and temperature values, we synthesize spatially and spectrally resolved X-ray observations with the XMM-Newton/EPIC-pn X-ray imaging spectrometers (Strüder et al. 2001). The method can be easily customized for other $\mathrm{X}$-ray instruments.

The emission measure in the $j$ th domain cell is calculated to be $\mathrm{em}_{\mathrm{j}}=n_{\mathrm{Hj}}^{2} V_{\mathrm{j}}$, where $n_{\mathrm{Hj}}^{2}$ is the particle number density in the cell, and $V_{\mathrm{j}}$ is the cell volume. We assume that the direction of the LoS corresponds to the $y$ axis (in the cartesian coordinate system), perpendicular to the direction of propagation of the SNR shock front, and that the depth along the LoS is $10 \mathrm{pc}$ (a typical value for the shells of evolved SNRs, such as in Vela and in Cygnus Loop). We then derive the distributions of emission measure versus temperature, $\operatorname{EM}(T)$, integrated along the LoS for each $(x, z)$, in the temperature range $5<\log T(\mathrm{~K})<7$ (divided into 50 bins, all equal on a logarithmic scale). From the $\operatorname{EM}(T)$ distributions, we synthesize maps of X-ray emission and $\mathrm{X}$-ray spectra, using the MEKAL spectral synthesis code (Mewe et al. 1985; Kaastra 1992; Kaastra \& Mewe 2000), assuming solar metal abundances (Grevesse \& Anders 1991).

We assume the source to be at a distance $D_{\text {snr }}=500 \mathrm{pc}$ (as, for instance, in the case of Cygnus Loop) and we filter the spectra through an ISM absorption column density, $N_{\mathrm{H}}=$ $5 \times 10^{20} \mathrm{~cm}^{-2}$ (Morrison \& McCammon 1983), according to typical values derived from SNR observations at that distance (e.g., Patnaude et al. 2002). The absorbed X-ray spectra are then folded through the instrumental response to obtain focal plane spectra. The exposure time is assumed to be $t_{\exp }=10 \mathrm{ks}$ for EPIC-pn (see, for instance, Miceli et al. 2005). The photon counts are randomized in each energy instrumental channel of the focal-plane spectra using Poisson statistics, by applying the rejection method to a Poisson distribution (Press et al. 1986). $\mathrm{X}$-ray emission maps are produced in selected energy bands, assuming a spatial resolution of 4 arcsec; the X-ray images are convolved with the corresponding point spread function (PSF), as given by Ghizzardi (2002) for EPIC-pn.

The final products are X-ray simulated observations, spatially and spectrally resolved, in a format virtually identical to that of real observations collected with EPIC-pn. To these data, we apply standard analysis methods commonly used for X-ray observations.

\section{Results}

\subsection{Lightcurves}

The detectability of the shock-cloud collision in the X-ray band is expected to depend on the $\mathcal{M}$ and $\chi$ parameters. From our simulations, we derive the X-ray lightcurves of the region associated with the shocked cloud, to understand at which stage of the interaction the visibility of the cloud is maximum. This region is selected in each synthesized EPIC-pn count-rate image in the [0.3-2.0] keV band (typically selected for the analysis of evolved SNR shock-cloud interaction; see, for instance, Miceli et al. 2005) by considering all the pixels with a median energy of X-ray photons, $\overline{\mathcal{E}}$ (Hong et al. 2004), which is less than $90 \%$ of the $\overline{\mathcal{E}}$ derived for the surrounding medium ${ }^{2}$. From these pixels, we then evaluate the average counts $\mathrm{s}^{-1}$ per pixel, $F_{\mathrm{X}}$, normalized to the value derived for the intercloud medium. The X-ray lightcurves reported in Fig. 2 indicate when the shocked cloud is detectable (when the normalized $F_{\mathrm{X}}$ is higher than 1) and its $\mathrm{X}$-ray luminosity is maximum.

In general, we find that the higher the value of $\mathcal{M}$, the higher the normalized $F_{\mathrm{X}}$ at each stage of the evolution (see Fig. 2). In most cases, the shocked cloud is visible in the X-ray band during the time interval $0.1 \tau_{\mathrm{cc}}<t<1.5 \tau_{\mathrm{cc}}$. The thermal conduction causes the shocked cloud to be brighter than in cases without conduction, broadening the peak in the X-ray lightcurve for any Mach number. The conduction contributes to the cloud heating, increasing the amount of cloud material above $1 \mathrm{MK}$ and emitting in the X-ray band. We also note that the conduction causes the shocked cloud to be hardly detectable in cases with $\chi \leq 3$

\footnotetext{
2 The shocked cloud material is expected to be cooler than the surrounding medium.
} 


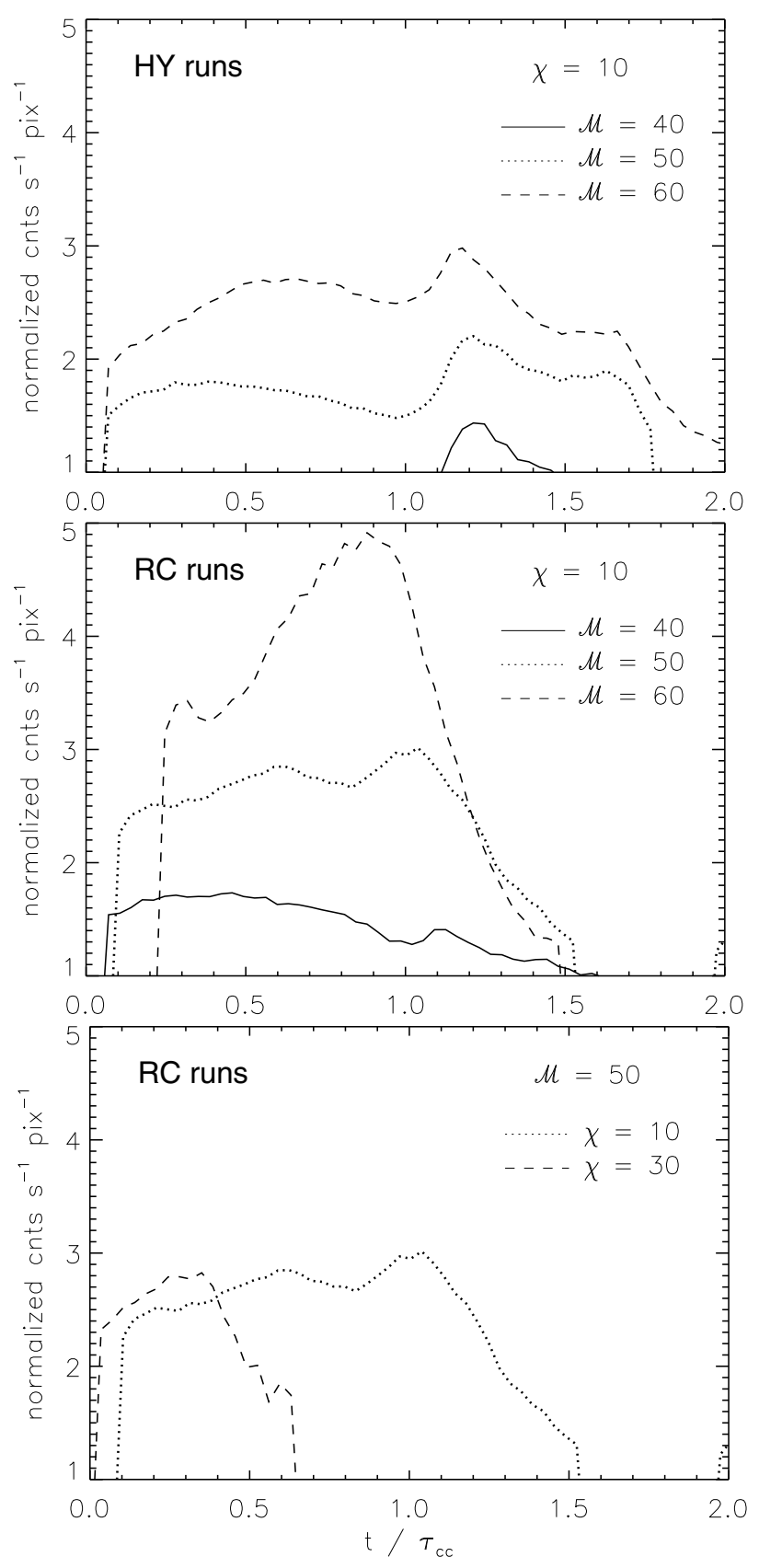

Fig. 2. X-ray lightcurves of the shock-cloud interaction region in the [0.3-2] keV band (see text for details on the definition). The timedependent surface brightness is normalized to the average value of the post-shock intercloud region. Upper panel: runs without thermal conduction (HY runs) with $\chi=10$ and $\mathcal{M}=40,50,60$. Middle panel: runs with thermal conduction (RC runs) with $\chi=10$ and $\mathcal{M}=40,50,60$. Lower panel: $\mathrm{RC}$ runs with $\mathcal{M}=50$ and $\chi=10,30$.

due to the quick evaporation of the cloud. For instance, in run $\mathrm{RCm} 50 \mathrm{c} 03$, there are no pixels with $\overline{\mathcal{E}}$ below $90 \%$ of the value derived for the background and no lightcurve can be reported in Fig. 2. On the other hand, the conduction produces shocked clouds with $\chi \gtrsim 20$, whose evolution is strongly dominated by the radiative losses, and are partially visible during the very early phases of the evolution (see lower panel in Fig. 2); these clouds would not be detectable in X-rays, in the absence of thermal conduction, because of their temperature $T_{\text {scl }} \approx 2.5 \times 10^{5} \mathrm{~K}$. In these cases, only the thermally conducting corona is detected, the core being much cooler than $1 \mathrm{MK}$.

\subsection{Spectral analysis}

The shocked cloud is detectable with EPIC-pn during the early phases of the shock-cloud interaction $\left(t<1.5 \tau_{\mathrm{cc}}\right.$; see Fig. 2$)$ as a bright knot surrounded by a diffuse region (see right panels in Fig. 3). We focus our spectral analysis on the time interval $0.4 \tau_{\mathrm{cc}} \leq t \leq 1.4 \tau_{\mathrm{cc}}$ when the shocked cloud reaches its highest luminosity in all the models. For each sampled X-ray image, we select spatial sub-regions in the computational domain and analyze the X-ray spectra extracted from each of them. To select spectrally homogeneous regions, we derive maps of the median energy of X-ray photons, $\overline{\mathcal{E}}$, from the EPIC-pn data that allows us to convey at the same time both spatial and spectral information about the emitting plasma (Hong et al. 2004). Since the shocked cloud is cooler than the surrounding medium, the median photon energy, $\overline{\mathcal{E}}$, of the bright region is lower than that of the surroundings (see left panels in Fig. 3). Thus we select subregions with a median photon energy of either $0.5 \mathrm{keV}<\overline{\mathcal{E}}<$ $0.6 \mathrm{keV}$ (to identify the knot) or $0.6 \mathrm{keV}<\overline{\mathcal{E}}<0.7 \mathrm{keV}$ (for the diffuse region, DR). The knot corresponds to the brightest portion of the X-ray image in Fig. 3, and the DR selects the intermediate brightness region surrounding the knot (compare the left and right panels of Fig. 3).

We emphasize that this definition of the extraction regions for spectral analysis is completely independent of the morphology of the X-ray emission. This has the great advantage that it can be straightforwardly applied to any current X-ray telescope observation for which the mean photon energy map can be computed. Moreover, it makes the spectral analysis independent, in first approximation, of the details of the shape of the ISM clouds, which in reality may be far more complex than the ideal spherical cloud proposed in our model.

The extracted spectra have a total number of photons ranging between $10^{4}$ and $4 \times 10^{5}$, which is adequate for performing a detailed spectral analysis. The focal plane spectra were analyzed using the spectral fitting package XSPEC (Arnaud 1996) and applying a multi-temperature fit to each spectrum. All the extracted spectra were closely fitted with two MEKAL components of an optically-thin thermal plasma in collisional ionization equilibrium (Mewe et al. 1985; Kaastra \& Mewe 2000), with solar abundances, and filtered through the interstellar absorption (Morrison \& McCammon 1983). We applied this procedure to any model listed in Table 1 , and in the interval $0.4 \tau_{\mathrm{cc}} \leq$ $t \leq 1.4 \tau_{\mathrm{cc}}$ in steps of $\delta t=0.1 \tau_{\mathrm{cc}}$. As an example, we present here the results obtained in the reference models HYm50c10 and $\mathrm{RCm} 50 \mathrm{c} 10$.

Figure 4 shows the temperature, $T$, and the emission measure per unit area, em $=\mathrm{EM} / A_{\text {reg }}$ (where $A_{\text {reg }}$ is the area of the selected region), of the isothermal components fitting the EPIC-pn spectra. When the thermal conduction is completely suppressed (run HYm50c10), the spectra of both the knot and the DR at the different epochs are described, in general, by two isothermal components with temperatures $T_{\text {low }} \approx 1 \mathrm{MK}$ and $T_{\text {high }} \approx 4.5 \mathrm{MK}$; the emission measure of the hot component is $\mathrm{em}_{\text {high }} \approx 5 \times 10^{18} \mathrm{~cm}^{-5}$ in all the spectra, whereas $\mathrm{em}_{\text {low }}$ ranges between $3 \times 10^{19}$ and $8 \times 10^{19} \mathrm{~cm}^{-5}$. We note that the temperature of the hot component is close to the temperature of the shocked ambient plasma $T_{\mathrm{psh}} \approx 4.7 \mathrm{MK}$, whereas $T_{\text {low }}$ is slightly higher than the temperature of the shock transmitted into the cloud, $T_{\mathrm{scl}} \approx 0.8 \mathrm{MK}$ (see Paper I). 
S. Orlando et al.: Observability and diagnostics in the X-ray band of shock-cloud interactions in SNRs
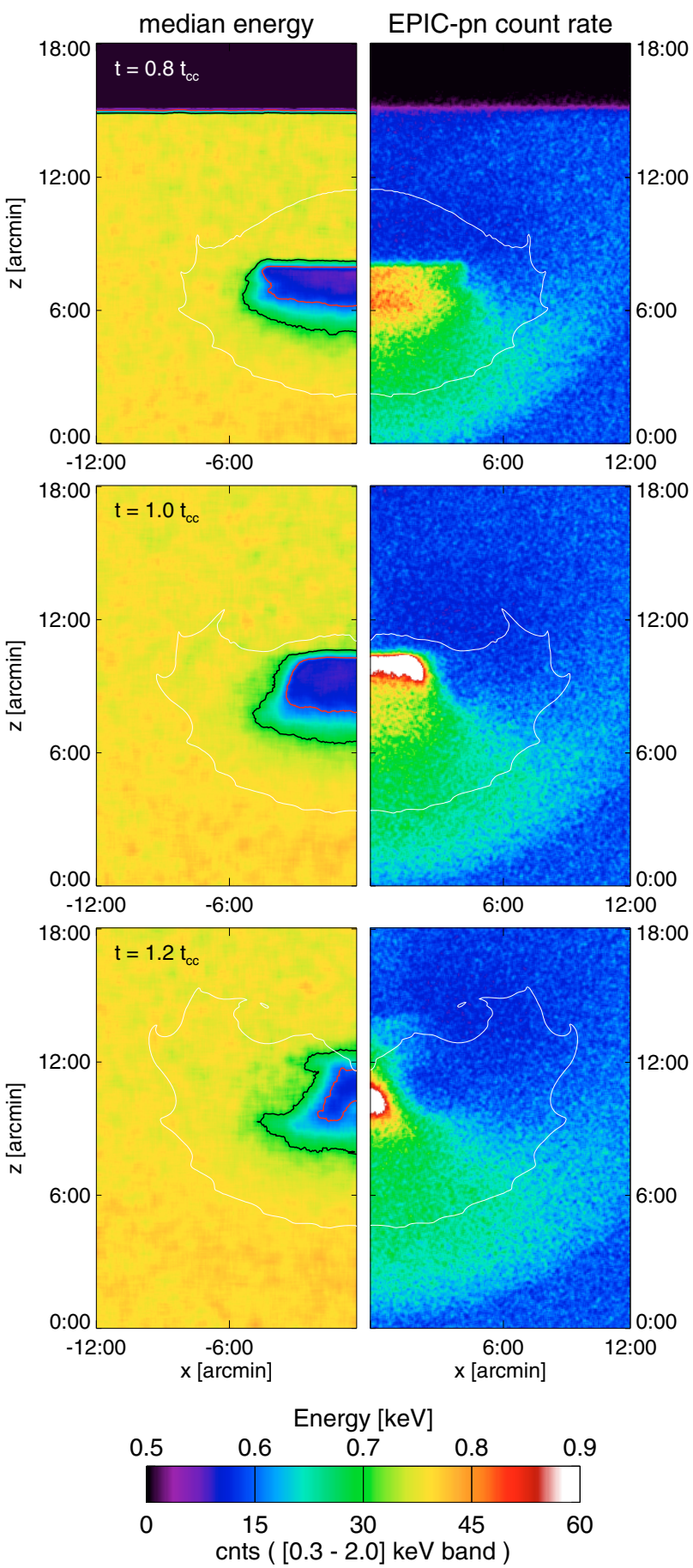

Fig. 3. Median energy maps (left) and EPIC-pn count-rate images (right) in the [0.3-2.0] $\mathrm{keV}$ band derived for run RCm50c10 at the three labeled times during the evolution. The pixel size is $\sim 4 \operatorname{arcsec}$ and the exposure time is $10 \mathrm{ks}$. The images are smoothed with a boxcar of width $\sigma=12$ arcsec. The white contours mark the cross-section of the cloud on the plane of the image, identified by zones consisting of the original cloud material by more than $90 \%$; the contours superimposed on the median energy maps mark the bright knot (red) and the diffuse region (DR; black).

In run $\mathrm{RCm} 50 \mathrm{c} 10$, the spectra are again described by two isothermal components, but with some differences caused by the thermal conduction. In particular, $T_{\text {low }}$ is higher and $T_{\text {high }}$ is lower than the values derived in HYm50c10, the difference being larger for the DR than for the knot. The value of $\mathrm{em}_{\text {low }}$ is
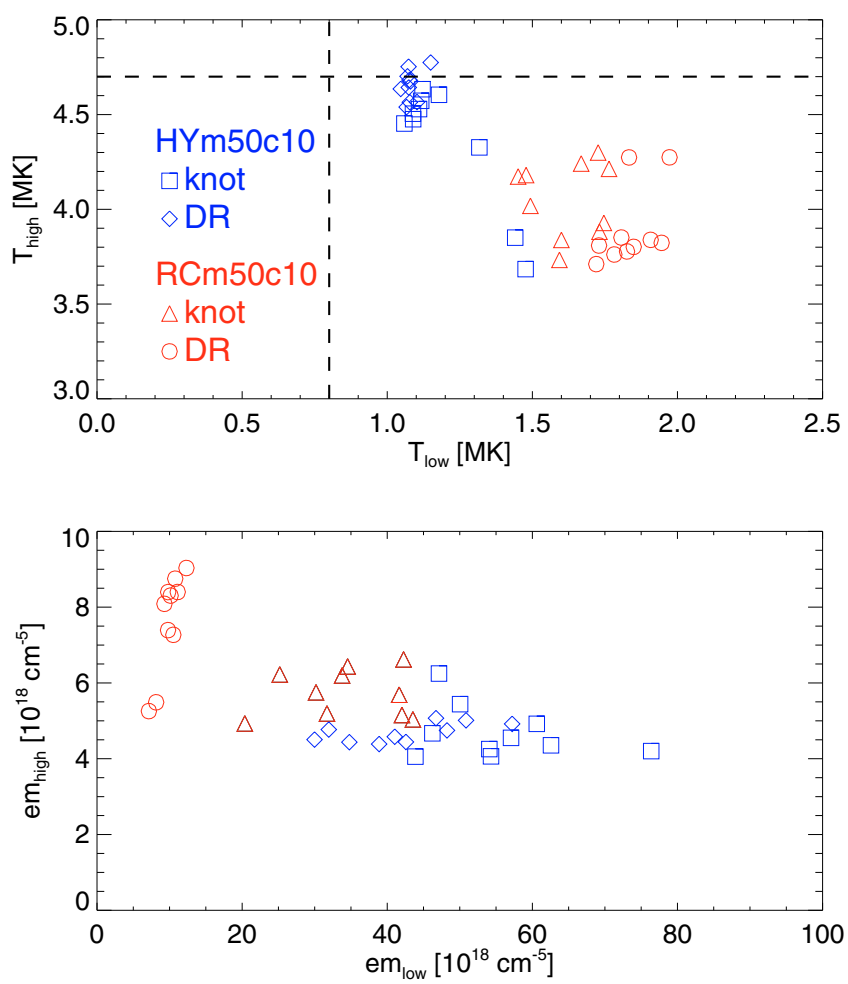

Fig. 4. Best-fit values of temperature (upper panel) and emission measure per unit area (lower panel) for the EPIC-pn spectra extracted from the bright knot and from the diffuse region (DR) in runs HYm50c10 (blue) and RCm50c10 (red) at different epochs between 0.4 and $1.4 \tau_{\mathrm{cc}}$. The dashed lines in the upper panel mark the temperatures expected for both the shock transmitted into the cloud $\left(T_{\text {scl }} \approx 0.8 \mathrm{MK}\right)$ and the shocked ambient plasma $\left(T_{\mathrm{psh}} \approx 4.7 \mathrm{MK}\right)$.

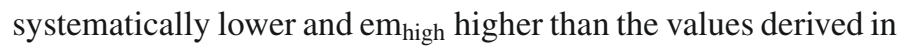
HYm50c10. In general, we find that the cold component is the most sensitive to thermal conduction, showing the largest differences between $\mathrm{HYm} 50 \mathrm{c} 10$ and $\mathrm{RCm} 50 \mathrm{c} 10$. The reasons for these differences can be understood by comparing the results of the spectral fitting with the distributions of emission measure per unit area versus temperature, $\operatorname{em}(T)$, in the region in which the extracted spectra originate.

Figure 5 shows these $\operatorname{em}(T)$ distributions and the results of the spectral fitting. In general, the distributions for both the knot and the DR are bimodal. The cold peak around $T \approx 2 \mathrm{MK}$ is produced by the shocked cloud gas; the hot peak at $T \approx 5 \mathrm{MK}$ is produced by the shocked ambient plasma surrounding the cloud. The best-fit values are localized around these maxima and can, therefore, be associated with both the shocked cloud gas (cold component) and the shocked ambient plasma (hot component).

In HYm50c10, the em( $T)$ distributions of both the knot and the DR do not change significantly during the evolution (except at $t \sim 1.2 \tau_{\mathrm{cc}}$, when the shocks transmitted from both the front and the rear of the cloud interact; see Paper II), the two bumps being steadily centered on the temperatures expected for the shock transmitted into the cloud $\left(T_{\text {scl }} \approx 0.8 \mathrm{MK}\right)$ and for the shocked ambient plasma ( $\left.T_{\mathrm{psh}} \approx 4.7 \mathrm{MK}\right)$, respectively. By comparing HYm50c10 with $\mathrm{RCm} 50 \mathrm{c} 10$, the main effects of the thermal conduction are: i) to smooth the $\mathrm{em}(T)$ distributions because of a transition region that forms between the inner part of the cloud and the ambient medium in which the density decreases and the temperature increases smoothly in the radial direction 


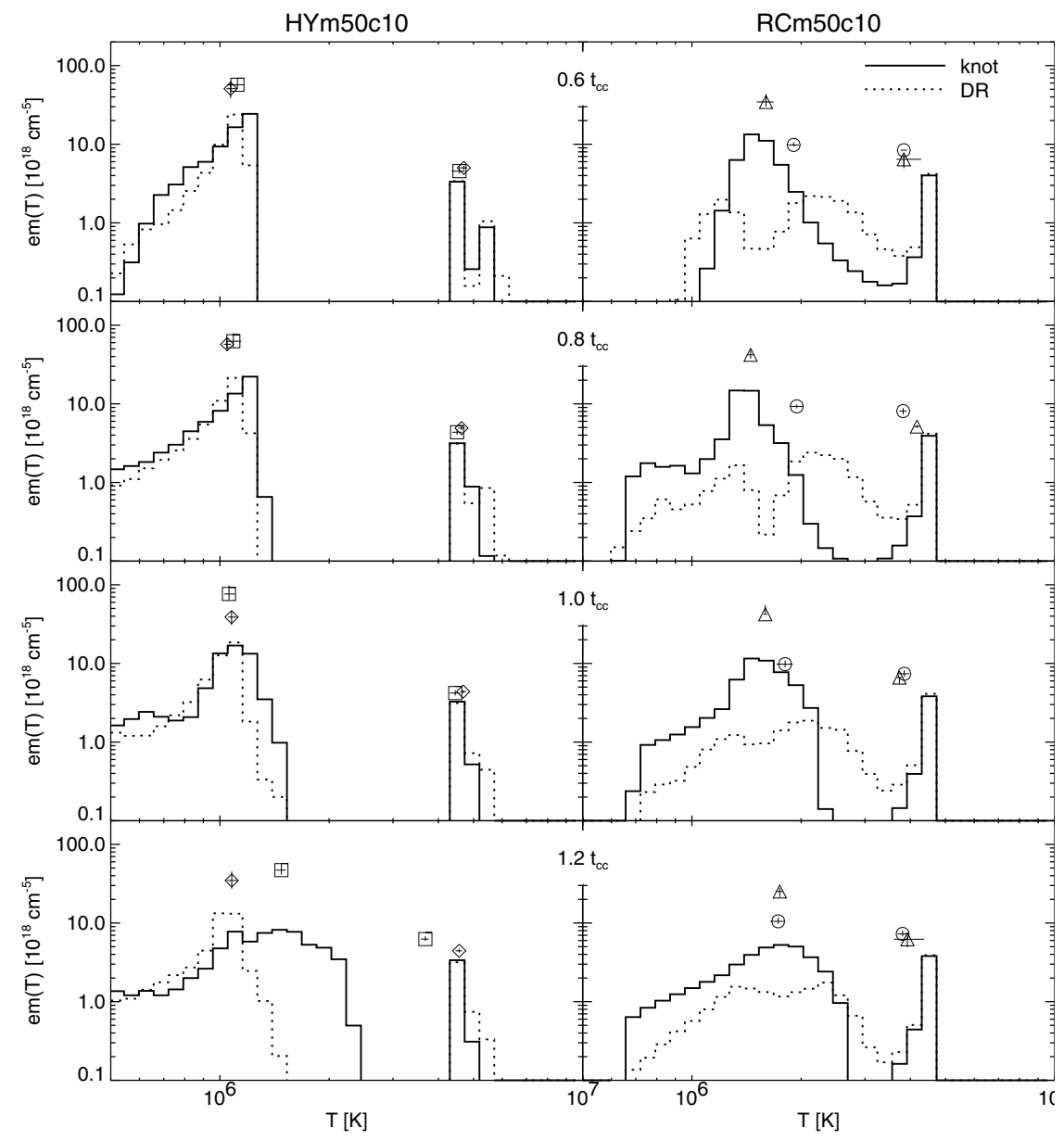

Fig. 5. Distributions of emission measure per unit area versus temperature, $\operatorname{em}(T)$, in the range of temperature $5<\log T(\mathrm{~K})<7$ for the knot (solid) and the diffuse region (DR, dotted) at the labeled times during the evolution. The figure shows the $\mathrm{em}(T)$ derived from both run HYm50c10 (on the left) and $\mathrm{RCm} 50 \mathrm{c} 10$ (on the right). The figure also shows the results of the spectral fitting for the EPIC-pn spectra extracted from the selected regions (symbols as in Fig. 4). The errors are at the $90 \%$ confidence level. (see Paper II); and ii) to shift the first bump to higher temperatures because of the gradual thermalization of the shocked cloud material to the temperature of the shocked ambient plasma (see also Paper II).

As a result of the conduction effects, the amount of plasma above $1 \mathrm{MK}$ increases in RCm50c10, making the shocked cloud brighter in the X-ray band (see Fig. 2). The changes in the em $(T)$ distributions caused by the thermal conduction also produce differences in the results of the spectral fitting for HYm50c10 and $\mathrm{RCm} 50 \mathrm{c} 10$ (see Fig. 4); for instance, the shift of the first bump in $\operatorname{em}(T)$ to higher temperatures leads to higher $T_{\text {low }}$, and the smoothing of em $(T)$ leads to lower em $\mathrm{em}_{\text {low }}$ in RCm50c10. We also note that the effects of the thermal conduction are greatest in the $\operatorname{em}(T)$ distribution of the DR, which represents the plasma of the corona surrounding the cloud core that is affected by efficient heat conduction. As a consequence, the cold fitting component in the DR is, in general, hotter than that in the knot (see also Fig. 4), whereas the opposite is true in HYm50c10 (i.e., the temperature of the shocked cloud material is never higher than the temperature of the shock transmitted into the cloud).

\section{Diagnostics}

\subsection{Median energy versus count-rate scatter plot}

Since the thermal conduction modifies the temperature and density structure of the shocked cloud (see Paper I), its effects should be evident in the comparison of $\overline{\mathcal{E}}$ maps (related to the spatial distribution of temperature) with count-rate maps (related to the spatial distribution of mass density). We derive, therefore, $\overline{\mathcal{E}}$ versus count-rate scatter plots (see, for instance, Miceli et al. 2005): we first divide the range of count-rate [0.01-0.20] cnts s ${ }^{-1}$ into 100 bins (all equal on linear scale); then, from the EPIC-pn count-rate images in the $[0.3-2.0] \mathrm{keV}$ band, we derive the median photon energy of all the pixels belonging to the same count-rate bin. Figure 6 shows the scatter plots derived for HY (upper panel) and RC (middle and lower panels) runs at selected epochs in the time interval $0.4 \tau_{\mathrm{cc}} \leq t \leq$ $1.4 \tau_{\mathrm{cc}}$ (when the shocked clouds are visible; see Fig. 2). All these plots are characterized by a clear descending trend and, then, in most cases, by a much flatter fall (cold plateau): the higher the count-rate, the lower the median energy and, therefore, the lower the average temperature along the LoS. The descending branch and the cold plateau are the signature of the shock-cloud collision: the former roughly corresponds to the DR and the latter to the bright and cold knot defined in Sect. 3.2.

In HY runs, scatter plots derived for $\chi=10$ and different $\mathcal{M}$ show a similar shape, characterized by a very steep descending branch and a well-defined cold plateau (see upper panel in Fig. 6). The descending branch shows an abrupt transition between the intercloud material (highest $\overline{\mathcal{E}}$ ) and the shocked cloud material (lowest $\bar{\varepsilon}$ ). In RC runs (see middle panel in Fig. 6), the thermal conduction makes the slope of the descending branch flatter than that of HY runs, the absolute value of the slope being smaller for higher $\mathcal{M}$. The flattening of the descending branch reflects a smooth temperature and density structure of the shocked cloud produced by the heat conduction, which causes the gradual growth of a transition region from the inner part of the cloud to the ambient medium (see Paper I). We note that the 
S. Orlando et al.: Observability and diagnostics in the X-ray band of shock-cloud interactions in SNRs
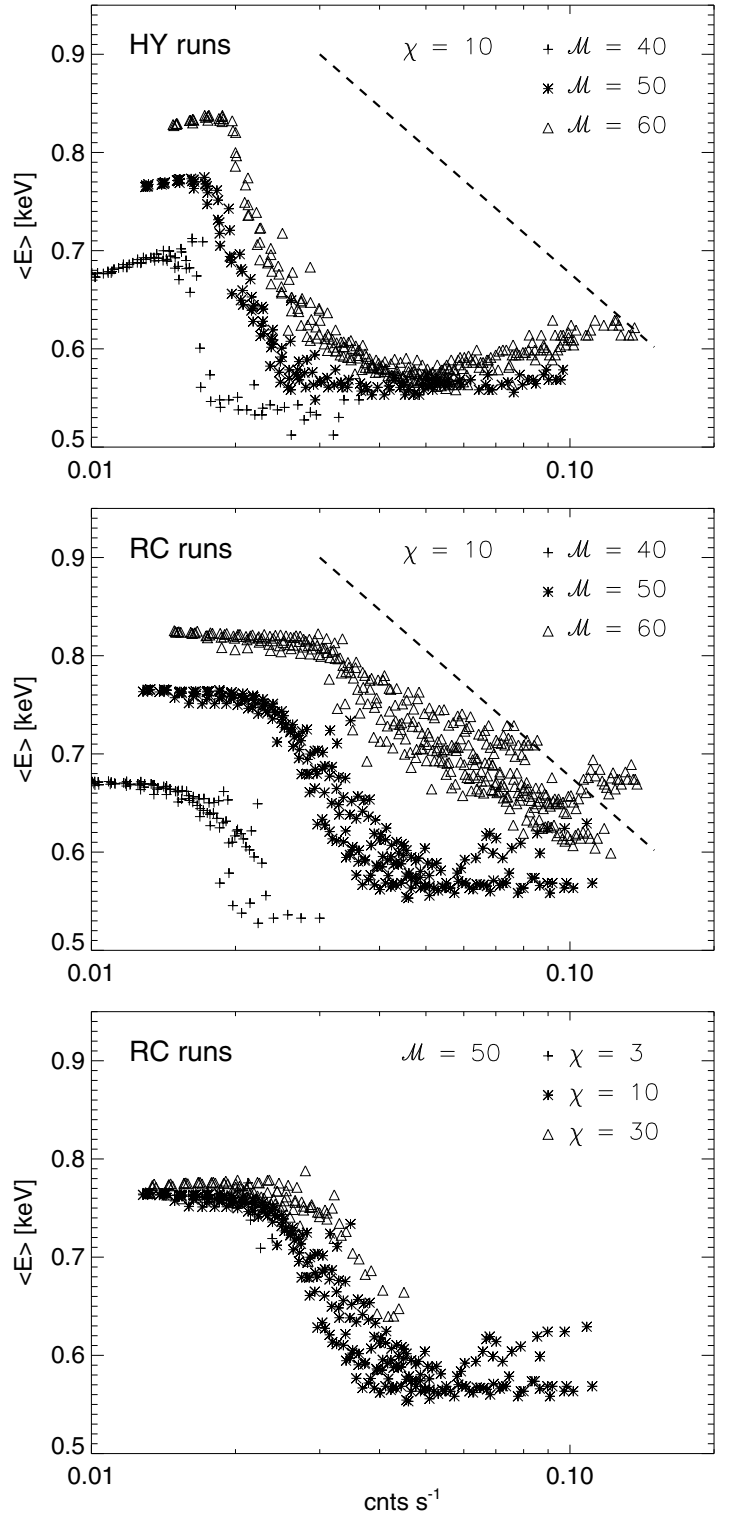

Fig. 6. Median photon energy, $\overline{\mathcal{E}}$, versus count-rate scatter plot derived from the EPIC-pn data in the period $0.4 \tau_{\mathrm{cc}} \leq t \leq 1.4 \tau_{\mathrm{cc}}$. Upper panel: runs without thermal conduction (HY runs) with $\chi=10$ and $\mathcal{M}=$ 40, 50,60. Middle panel: runs with thermal conduction (RC runs) with $\chi=10$ and $\mathcal{M}=40,50,60$. Lower panel: $\mathrm{RC}$ runs with $\mathcal{M}=50$ and $\chi=3,10,30$. The dashed lines in the upper and middle panels show the slope of the $\bar{\varepsilon}$ versus count-rate scatter plot derived from the analysis of EPIC data of Vela FilD (Miceli et al. 2005).

scatter plots of runs with different $\chi$ but identical $\mathcal{M}=50$ are virtually indistinguishable (see lower panel in Fig. 6) independently of the role of either radiation or conduction. This is because the contribution of the shocked cloud to X-ray emission originates invariably in regions dominated by thermal conduction. In particular, the X-ray emission in run $\mathrm{RCm} 50 \mathrm{c} 30$ originates in the thermally conducting corona, the cloud core being at temperatures $T \lesssim 2 \times 10^{5} \mathrm{~K}$.

In summary, these scatter plots can be very useful for determining the role of thermal conduction through the slope of the descending branch and inferring the speed of the shock when there is efficient conduction. On the other hand, the plots are poorly useful for inferring the true density contrast of the cloud.

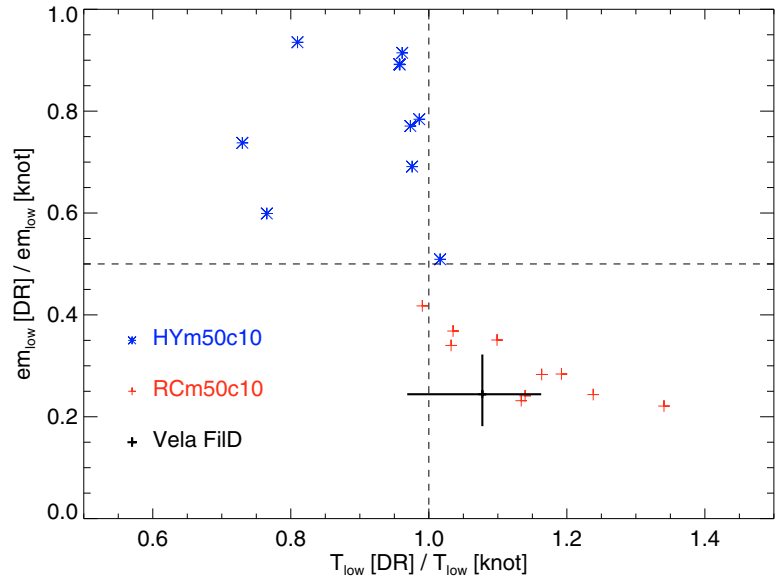

Fig. 7. The figure compares the temperature and the emission measure per unit area of the cold component derived for the knot with those derived for the diffuse region (DR). The blue stars (red crosses) mark the values derived in run HYm50c10 (RCm50c10) and reported in Fig. 4; the black symbol with error bars mark the values derived from the analysis of EPIC data of Vela FilD (regions 4, knot, and 1, diffuse region, in Miceli et al. 2005).

\subsection{Temperature and emission measure ratios}

The spectral analysis discussed in Sect. 3.2 implies that the cold fitting components describing the knot and the DR are sensitive to thermal conduction; we therefore propose to use them as a diagnostic tool for tracing the efficiency of conduction. Figure 7 compares the temperature and emission measure values derived for the knot with those derived for the DR (and reported in Fig. 4) in runs HYm50c10 and RCm50c10. The runs either with or without thermal conduction can be clearly separated in the plot, the thermal conductive case being localized in the bottomright quadrant and the pure hydrodynamic case in the top-left quadrant. This result is determined by the development of the thermally conducting corona in RCm50c10 and is therefore expected to be general. In particular, as discussed in Sect. 3.2, the thermal conduction smooths the first bump in the em $(T)$ distributions and shifts it to higher temperatures, this effect being larger for the DR than for the knot. As a result, the cold component fitted for the DR is, in general, hotter than that derived for the knot (i.e., $T_{\text {low }}[\mathrm{DR}]>T_{\text {low }}[\mathrm{knot}]$ ), whereas the opposite is true (i.e., $T_{\text {low }}[\mathrm{DR}]<T_{\text {low }}[\mathrm{knot}]$ ) if the conduction is suppressed. The smoothing of the $\operatorname{em}(T)$ distribution due to the conduction is also the largest for the DR (because the corona surrounding the cloud core is subject to efficient heat conduction), leading to the smallest values of $\mathrm{em}_{\text {low }}$ (see also lower panel in Fig. 4).

In summary, the temperature and emission measure ratios are an excellent way of determining the role of the thermal conduction in the evolution of the system.

\subsection{An example of model versus observation comparison}

Our study shows that evidence of thermal conduction at work during the shock-cloud interaction can be found in the spectral analysis of X-ray data. As discussed in the previous sections, the $\overline{\mathcal{E}}$ versus count-rate scatter plot and the temperature and emission measure ratios can be efficient diagnostic tools for deriving the shock speed and the role of thermal conduction, which, in turn, is linked to the magnetic field configuration, as shown by Orlando et al. (2008). In this section, we challenge the above diagnostic tools and show that they can easily be used in the 
analysis of X-ray data, by comparing our model results with Xray observations reported in the literature.

In particular, we focus on a well-studied region, the "FilD region", that is an isolated, bright X-ray knot in the northern rim of the Vela SNR. Because of its proximity ( $250 \mathrm{pc}$, Bocchino et al. 1999; Cha et al. 1999), Vela is an ideal target for this kind of study, allowing us to observe the interaction of the SNR shock front with relatively small clouds, such as FilD $\left(\sim 2 \times 10^{18} \mathrm{~cm}\right.$; see Miceli et al. 2005) in great detail. The analysis of an XMMNewton observation of FilD (Miceli et al. 2005) demonstrated that its X-ray spectra can be modeled as that of an optically-thin plasma with two thermal components (at $\sim 1 \mathrm{MK}$ and $\sim 3 \mathrm{MK}$, respectively) with inhomogeneous volume distributions along the line of sight. The cold component dominates in the brightest region that is surrounded by a diffuse region with harder X-ray emission. To interpret these results, Miceli et al. (2006) developed a detailed hydrodynamic model of FilD, synthesized X-ray emission maps, and spectra from the model, and compared them with the data. Their analysis showed that the X-ray and optical emission of FilD can be explained as the result of the interaction of a SNR shock (with Mach number $\mathcal{M}=57$ ) with an ellipsoidal cloud 30 times denser than the intercloud medium, the estimated interaction time being $\sim 0.32 \tau_{\mathrm{cc}}$. Miceli et al. (2006) proved that the two components originate in the cloud material heated by both the transmitted shock front and heat conduction between the cloud and the hotter, shocked intercloud medium. FilD, therefore, is an ideal benchmark for our model, because it is a case in which the thermal conduction is clearly at work. Since the parameters used in our simulations differ slightly from the parameters deduced from the observations (including the shape of the cloud), we do not expect a perfect match, but the comparison will nonetheless provide us with much useful information.

Among the runs presented here, the one matching the density contrast of the shock-cloud interaction is RCm50c30 (see Table 1). As already discussed, the shocked cloud with this density contrast $(\chi \sim 30)$ would not be detectable in $X$-rays if the thermal conduction is suppressed, its estimated temperature being $T_{\mathrm{scl}} \approx 3.5 \times 10^{5} \mathrm{~K}$. On the other hand, after inspecting Fig. 2, we note that, in run $\mathrm{RCm} 50 \mathrm{c} 30$ (dashed line in the lower panel), the shocked cloud is visible in X-rays for a short time interval $\left(0.1-0.6 \tau_{\mathrm{cc}}\right)$ and is of the highest surface brightness during the evolutionary stage estimated for FilD $\left(\sim 0.32 \tau_{\mathrm{cc}}\right)$. We expect to detect brighter emission for higher values of $\mathcal{M}$ (see middle panel in Fig. 2). As shown by our simulations, the detected X-ray emission originates in the cloud material dominated by thermal conduction, confirming the relevance of conduction in the evolution of FilD.

Miceli et al. (2005) analyzed the spectra extracted from the knot and the DR contained by the FilD region. Thus, we can derive the temperature and emission measure ratios of the cold components derived by these authors and plot them in Fig. 7. The observed values lie in the bottom-right quadrant of the figure, confirming once again that in FilD the thermal conduction is efficient, in perfect agreement with the independent conclusion of Miceli et al. (2006). The diagnostics presented in Fig. 7 can be easily implemented and we propose their use as a standard for verifying the role of conduction.

Miceli et al. (2005) also derived a $\overline{\mathcal{E}}$ versus count-rate scatter plot for FilD that can be directly compared with the corresponding scatter plots derived with our models. We overplot a best-fit power-law model (with index $=-0.25$ ) onto the data of the FilD region reported in Fig. 4 of Miceli et al. (2005), considering the count-rate as a free parameter, a reasonable choice if we consider that the true value of the count-rate depends on the actual LoS extension, which is poorly known. The slope of the observed scatter plot is rather flat and cannot be reproduced by models without thermal conduction (see upper panel of Fig. 6). On the other hand, the observed slope is reproduced quite well by our RC runs, in agreement with the evidence that the thermal conduction plays an important role in the evolution of FilD. The model with $\mathcal{M}=60$ appears to be the most successful in reproducing the slope, in very good agreement with the value obtained by Miceli et al. (2005) $(\mathcal{M}=57)$ with a detailed analysis.

\subsection{Limits of the model}

In our simulations, we parametrize the thermal conductivity using the classical Spitzer's conductivity and the saturation limit, by assuming essentially laminar thermal conduction across the entire spatial domain. However, regions of strong turbulence of different strength and extent can develop in the system (especially in shock-cloud interactions dominated by radiative cooling), for instance at the shear layers along the cloud boundary or at the vortex sheets in the cloud wake. The turbulence in these regions may have a significant effect on the thermal conduction, leading to significant deviations of thermal conductivity from its laminar values (e.g., Kulsrud 1983; Narayan \& Medvedev 2001; Lazarian 2006). As a result of the turbulence, the thermal conduction may be inhomogeneous. On the other hand, the deviations of thermal conductivity from laminar values are expected to be relevant in the shocked intercloud medium, and thus should not affect our main conclusions about the effects of thermal conduction on the shocked cloud and the applicability of the diagnostics developed here.

In our model, we do not account for the possible effect of the back-reaction of accelerated cosmic rays on shock dynamics. In the case of high Mach number shocks, a part of the shock power may be dissipated into cosmic-ray acceleration, resulting in an increase in the shock compression ratio. The distribution function of non-thermal particles and the bulk flow profile for the shock upstream region are sensitive to the total compression ratio. Thus, even a moderate efficiency of particle acceleration may reduce the post-shock ion and electron temperatures (see, e.g., Eq. (18) in Bykov et al. 2008), which would have implications for the X-ray emission. This effect is expected to be large for shocks with high Mach number (as, for instance, in young SNRs), but not for middle-aged SNRs (on which this paper is focused) for which no non-thermal emission has been detected. Even if particle acceleration were not negligible, the relevant effect on our diagnostics would only be to slightly reduce the efficiency of the thermal conduction because of the lower postshock temperature. As in the case of magnetized clouds (Orlando et al. 2008), shocked clouds with considerable particle acceleration would fall in-between the limit of completely suppressed thermal conduction (HY runs) and the unmagnetized limit of conduction (RC runs) discussed in this paper.

Pittard et al. (2009) demonstrated that turbulence plays an important role in shock-cloud interactions, and that environmental turbulence adds a new dimension to the parameter space. In particular, these authors showed that turbulence is generated mainly around the cloud boundary and in the cloud wake after $\sim \tau_{\mathrm{cc}}$, the main effect being that clouds subject to a highly turbulent post-shock environment are destroyed significantly more rapidly than those within a smooth flow: the greater the cloud density contrast $\chi$, the higher the effect of turbulence (for instance, for $\chi \approx 100$, the effect of the post-shock turbulence dominates the shock-cloud interaction). On the other hand, an 
efficient thermal conduction smooths the cloud boundary very quickly (see Paper I), and turbulence grows more slowly around clouds with a smooth density profile (Pittard et al. 2009). Thus we are confident that our results are valid and the diagnostics proposed here can be applied to clouds of moderate density contrast (i.e., the effects of turbulence poorly influence the shockcloud interaction at early evolutionary stages for $t<\tau_{\mathrm{cc}}$ ) and smooth density profiles (i.e., the growth of turbulence around clouds is slow), which we have considered here.

Finally, our model does not account for the incomplete electron-ion temperature equilibration in the post-shock region. Equilibrium may not be completed early during the shock-cloud interaction $\left(t \approx 0.1 \tau_{\mathrm{cc}}\right)$. We should apply emission models including non-equilibrium in that phase and the initial part of the lightcurves presented in Fig. 2 may be subject to change. On the other hand, the diagnostics discussed in this section refer to the shock-cloud interaction at $0.4 \tau_{\mathrm{cc}} \leq t \leq 1.4 \tau_{\mathrm{cc}}$, when the hypothesis of temperature equilibration can be considered realistic for the shock velocities explored here (Rakowski et al. 2003).

\section{Summary and conclusion}

In a series of previous papers (Papers I, II, Orlando et al. 2008), we have investigated the $\mathrm{X}$-ray emission originating from the interaction of SNR shock waves with isolated gas clouds with the aim of identifying the plasma structures that contribute primarily to X-ray emission detectable with current X-ray instruments. In this work, we have extended the parameter space previously studied considering clouds with different values of density contrast and developing diagnostics in the X-ray band to derive the shock speed, which is one of the fundamental parameter governing the shock-cloud interactions, and the cloud evaporation under the effect of the thermal conduction.

In particular, by performing a series of spectral fit to the simulated data of the shock-cloud interaction region, we have proven that there are at least two interesting diagnostic diagrams that can be used:

- the median energy versus count-rate scatter plot (Fig. 6), which provides a direct estimate of the shock speed and insight into the effects of the thermal conduction;

- the temperature and emission measure ratios of the knot to the diffuse region of the cloud (Fig. 7), which provide direct estimates of the role of the thermal conduction in the evolution of the system.

We emphasize that the regions (the knot and the DR) that must be selected to derive the diagnostic diagrams are defined entirely on the basis of mean photon energy maps, and not on the shape of the X-ray emission. Therefore, the method is very well-posed and independent, to a first approximation, of the true shape of the ISM clouds, which could be more complex than the ideal spherical cases considered in our hydrodynamic simulations. While this method cannot be considered a substitution for a detailed approach to the study of shock-cloud interactions based on the development of ad-hoc (and time-consuming) numerical models, we have nevertheless demonstrated that the diagnostic diagrams that we have presented can be very useful in determining some of the parameters of the system and the role of thermal conduction in a very quick and straightforward way. These pieces of information can in turn be used for a more detailed model, if necessary.

The method can be applied to imaging X-ray observations of middle-aged thermal SNR shells (like Vela or Cygnus Loop), such as those obtained by the XMM-Newton and Chandra X-ray satellites. We used as a benchmark the XMM-Newton/EPIC observations of the Vela FilD region of Miceli et al. (2005), from which, independently, Miceli et al. (2006) found strong evidence of thermal conduction at work during the shock-cloud interaction, using a detailed ad-hoc numerical model. We found that our method is quite effective in quickly recovering the shock speed and the effects of the thermal conduction.

Acknowledgements. We thank the referee for constructive and helpful criticism. The software used in this work was in part developed by the DOEsupported ASC / Alliance Center for Astrophysical Thermonuclear Flashes at the University of Chicago. The simulations have been executed at CINECA (Bologna, Italy) in the framework of the INAF-CINECA agreement on "High Performance Computing resources for Astronomy and Astrophysics", and on the SCAN (Sistema di Calcolo per l'Astrofisica Numerica) HPC facility of the INAF - Osservatorio Astronomico di Palermo. This work was supported in part by Ministero dell'Università e della Ricerca and by Istituto Nazionale di Astrofisica.

\section{References}

Arnaud, K. A. 1996, in Astronomical Data Analysis Software and Systems V, ASP Conf. Ser., 101, 17

Bocchino, F., Maggio, A., \& Sciortino, S. 1999, A\&A, 342, 839

Bocchino, F., Maggio, A., Sciortino, S., \& Raymond, J. 2000, A\&A, 359, 316

Borkowski, K. J., Shull, J. M., \& McKee, C. F. 1989, ApJ, 336, 979

Bykov, A. M., Dolag, K., \& Durret, F. 2008, Space Sci. Rev., 134, 119

Cha, A. N., Sembach, K. R., \& Danks, A. C. 1999, ApJ, 515, L25

Cowie, L. L., \& McKee, C. F. 1977, ApJ, 211, 135

Fadeyev, Y. A., Le Coroller, H., \& Gillet, D. 2002, A\&A, 392, 735

Fryxell, B., Olson, K., Ricker, P., et al. 2000, ApJS, 131, 273

Ghizzardi, S. 2002, In Flight Calibration of the PSF for the pn Camera, XMM-SOC-CAL-TN-0029

Giuliani, J. L. 1984, ApJ, 277, 605

Graham, J. R., Levenson, N. A., Hester, J. J., Raymond, J. C., \& Petre, R. 1995, ApJ, 444, 787

Grevesse, N., \& Anders, E. 1991, Solar element abundances, Solar interior and atmosphere, A92-36201 14-92 (Tucson, AZ: University of Arizona Press), 1227

Hong, J., Schlegel, E. M., \& Grindlay, J. E. 2004, ApJ, 614, 508

Kaastra, J. S. 1992, An X-Ray Spectral Code for Optically Thin Plasmas, Internal SRON-Leiden Report, updated version 2.0

Kaastra, J. S., \& Mewe, R. 2000, in Atomic Data Needs for X-ray Astronomy, 161

Klein, R. I., McKee, C. F., \& Colella, P. 1994, ApJ, 420, 213

Kulsrud, R. M. 1983, Handbook of plasma physics, 1, ed. A. A. Galeev, \& R. N. Sudan (Amsterdam - New York - Oxford: North-Holland Publishing Company), 115

Lazarian, A. 2006, ApJ, 645, L25

MacNeice, P., Olson, K. M., Mobarry, C., de Fainchtein, R., \& Packer, C. 2000, Comp. Phys. Comm., 126, 330

Mewe, R., Gronenschild, E. H. B. M., \& van den Oord, G. H. J. 1985, A\&AS, 62,197

Miceli, M., Bocchino, F., Maggio, A., \& Reale, F. 2005, A\&A, 442, 513

Miceli, M., Reale, F., Orlando, S., \& Bocchino, F. 2006, A\&A, 458, 213

Morrison, R., \& McCammon, D. 1983, ApJ, 270, 119

Narayan, R., \& Medvedev, M. V. 2001, ApJ, 562, L129

Nichols, J. S., \& Slavin, J. D. 2004, ApJ, 610, 285

Orlando, S., Peres, G., Reale, F., et al. 2005, A\&A, 444, 505 (Paper I)

Orlando, S. Bocchino, F., Peres, G., et al. 2006, A\&A, 457, 545 (Paper II)

Orlando, S., Bocchino, F., Reale, F., Peres, G., \& Pagano, P. 2008, ApJ, 678, 274

Patnaude, D. J., Fesen, R. A., Raymond, J. C., et al. 2002, AJ, 124, 2118

Pittard, J. M., Falle, S. A. E. G., Hartquist, T. W., \& Dyson, J. E. 2009, MNRAS, 394, 1351

Press, W. H., Flannery, B. P., Teukolsky, S. A., \& Vetterling, W. T. 1986,

Numerical Recipes (Cambridge: Cambridge University Press)

Rakowski, C. E., Ghavamian, P., \& Hughes, J. P. 2003, ApJ, 590, 846

Raymond, J. C., \& Smith, B. W. 1977, ApJS, 35, 419

Spitzer, L. 1962, Physics of Fully Ionized Gases (New York: Interscience)

Strüder, L., Briel, U., Dennerl, K., et al. 2001, A\&A, 365, L18

Zel'dovich, Y. B., \& Raizer, Y. P. 1966, Physics of Shock Waves and HighTemperature Hydrodynamic Phenomena (New York: Academic Press) 